\title{
Selection of Efficient Parameter Estimation Method for Two-Parameter Weibull Distribution
}

\author{
Mohammed M. A. Almazah $\mathbb{D}^{1,2}$ and Muhammad Ismail $\mathbb{1}^{3}$ \\ ${ }^{1}$ Mathematics Department, College of Sciences and Arts (Mayhill), King Khalid University, Abha, Saudi Arabia \\ ${ }^{2}$ Mathematics and Computer Department, College of Sciences, Ibb University, Lapai, Yemen \\ ${ }^{3}$ Department of Statistics, COMSATS University, Lahore Campus, Lahore, Pakistan \\ Correspondence should be addressed to Mohammed M. A. Almazah; mmalmazah@kku.edu.sa and \\ Muhammad Ismail; drismail39@gmail.com
}

Received 27 July 2021; Revised 3 September 2021; Accepted 22 October 2021; Published 2 November 2021

Academic Editor: Mauro Gaggero

Copyright (c) 2021 Mohammed M. A. Almazah and Muhammad Ismail. This is an open access article distributed under the Creative Commons Attribution License, which permits unrestricted use, distribution, and reproduction in any medium, provided the original work is properly cited.

\begin{abstract}
Several studies have considered various scheduling methods and reliability functions to determine the optimum maintenance time. These methods and functions correspond to the lowest cost by using the maximum likelihood estimator to evaluate the model parameters. However, this paper aims to estimate the parameters of the two-parameter Weibull distribution $(\alpha, \beta)$. The maximum likelihood estimation method, modified linear exponential loss function, and Wyatt-based regression method are used for the estimation of the parameters. Minimum mean square error (MSE) criterion is used to evaluate the relative efficiency of the estimators. The comparison of the different parameter estimation methods is conducted, and the efficiency of these methods is observed, both mathematically and experimentally. The simulation study is conducted for comparison of samples sizes (10, 50, 100,150 ) based on the mean square error (MSE). It is concluded that the maximum likelihood method was found to be the most efficient method for all sample sizes used in the research because it achieved the least MSE compared with other methods.
\end{abstract}

\section{Introduction}

The Weibull distribution is the continuous probability distribution. It has been frequently used as a failure model in reliability studies to describe all phases of machine failure $[1,2]$. Waloddi Weibull used the probabilistic Weibull model to indicate the distribution of the breaking strength of materials and to illustrate the performance of systems or events that have some degree of variability [3]. It has frequently been utilized to evaluate the reliability of the product, examine life data, and model failure times [4].

The Weibull distribution is commonly utilized in radar systems to model the variation of the signal level triggered by clutters. It is also used in wireless communications [4]. Further, the Weibull distribution has significant importance in reliability and life testing. It is the most frequently used distribution in the reliability theory. When employed, its greater effects generate more accurate results and have crucial applications for studying failure models [5]. Several studies have used this distribution for the analysis [4, 6-8]. Hallinan [9] gave a perceptive appraisal by introducing numerous chronological facts and various characteristics of this distribution. A detailed chapter on the systematic behavior of this distribution is contributed by Coleman et al. [10]. More recently, Lai et al. [2] wrote a monograph that comprises all aspects concerning the Weibull distribution and its expansions. Moreover, for the relevant information and details on applications, we recommend the readers to read several studies proposed in $[4,6,8,11-14]$. The Bayesian estimation is commonly used in various scientific areas [15-17]. There exists much literature on the subject; due to the limitations of the paper, we are incapable of relating all related information.

The previous paper [3] uses scheduling techniques and reliability function to establish the optimum maintenance time, which corresponds to the lowest cost, by employing the maximum likelihood estimator (MLE) to estimate model 
parameters. However, the current study is initiated to estimate the scale parameter $(\alpha)$ of the two-parameter Weibull distribution $(\alpha, \beta)$ in a mathematical method and obtain an estimator that comprises a minimum mean square error (MSE) and determines the relative efficiency of the estimators and comparability of estimators.

\section{Materials and Methods}

2.1. The Two-Parameter Weibull Distribution. The Weibull distribution contains one, two, or three parameters. These parameters are shape parameter $(\beta)$ and scale parameter $(\alpha)$, and there may be a third parameter, the location $(\gamma)$ parameter [5]. The distribution function of two-parameter Weibull distribution can be expressed as follows:

$$
f(t)=\beta \alpha^{-\beta} t^{\beta-1} e^{-(t / \alpha)^{\beta}}, \quad \beta>0, \alpha>0, t>0,
$$

where

(1) $\alpha$ is considers as the scale parameter, which is recognized as the Weibull slope and takes a positive value.

(2) $\beta$ is considered as the shape parameter. It defines the form of distribution and takes a positive value.

(3) $\gamma$ is the location parameter $(\gamma=0)$.

The cumulative distribution function (CDF) can be written as follows:

$$
F(t)=1-e^{-(t / \alpha)^{\beta}}
$$

and the survival function can be written as follows:

$$
R(t)=1-F(t)=e^{-(t / \alpha)^{\beta}},
$$

and the failure function for the two-parameter Weibull distribution can be written as follows:

$$
h(t)=\frac{f(t)}{R(t)}=\beta \alpha^{-\beta} t^{\beta-1},
$$

where

(1) $\beta>1$ means the failure rate is increasing.

(2) $\beta<1$ means the failure rate is decreasing.

(3) $\beta=1$ means fixed failure rate.

The $r^{\text {th }}$ moment of the Weibull distribution is

$$
M_{r}=\alpha^{r} \Gamma\left(\frac{r}{\beta}+1\right)
$$

and when $r=1,2$, we get the first and second moments $M_{1}=\alpha \Gamma(1 / \beta+1)$ and $M_{2}=\alpha^{2} \Gamma(2 / \beta+1)$.

Thus, the mean and variance of the two-parameter Weibull distribution are, respectively,

$$
\begin{aligned}
\text { Mean } & =\alpha \Gamma\left[1+\frac{1}{\beta}\right], \\
\operatorname{Var} & =\alpha^{2}\left[\Gamma\left\{\frac{\beta+2}{\beta}\right\}\right]-\left[\Gamma\left\{\frac{\beta+1}{\beta}\right\}\right]^{2} .
\end{aligned}
$$

The standard $=\sqrt{\alpha^{2}[\Gamma\{\beta+2 / \beta\}]-[\Gamma\{\beta+1 / \beta\}]^{2}}$ deviation
The reliability function can be written as

$$
R(t)=e^{-(t / \alpha)^{\beta}} \text {. }
$$

2.2. The Parameter Estimation of the Weibull Distribution. In the following subsections, we describe different parameter estimation methods for Weibull distribution.

2.2.1. Maximum Likelihood Estimation (MLE). MLE is the most commonly used method for the estimation of parameters. It makes the possible function based on the maximum. It demonstrates the function of the maximum likelihood, so the most plausible results can be obtained from relationship (1) by the following equation:

$$
L\left(t_{i}, \beta, \alpha\right)=\prod_{i=1}^{n} f\left(x_{i}, \beta, \alpha\right)=\frac{\beta^{n}}{\alpha^{n}} \prod_{i=1}^{n}\left(\frac{t}{\alpha}\right)^{\beta-1} e^{\sum_{i=1}^{n}(t / \alpha)^{\beta}} .
$$

Equation (8) can be linearized by taking the logarithm on both sides:

$$
\ln L\left(t_{i}, \beta, \alpha\right)=n \ln \beta-n \ln \alpha+(\beta-1) \sum_{i=1}^{n} \ln \left(\frac{t_{i}}{\alpha}\right)-\sum_{i=1}^{n}\left(\frac{t_{i}}{\alpha}\right)^{\beta} .
$$

MLE of the parameter is the value of the parameter that maximizes $L$ (likelihood). MLE for the Weibull distribution can be obtained by solving the equations resulting from setting the two partial derivatives of $L(\alpha, \beta)$ to zero:

$$
\begin{aligned}
\frac{n}{\widehat{\beta}}+\sum_{i=1}^{n} \ln t_{i}-\frac{1}{\alpha} \sum_{i=1}^{n} t_{i}^{\beta} \ln t_{i} & =0, \\
\frac{-n}{\alpha}+\frac{1}{\alpha^{2}} \sum_{i=1}^{n} t_{i}^{\beta} & =0 .
\end{aligned}
$$

Then, $\widehat{\beta}$ is the solution of

$$
\frac{1}{\widehat{\beta}}+\frac{1}{n} \sum_{i=1}^{n} \ln t_{i}-\frac{\sum_{i=1}^{n} t_{i}^{\beta} \ln t_{i}}{\sum_{i=1}^{n} t_{i}^{\beta}}=0 .
$$

Traditional methods cannot be used to solve equation (11). We can use the Newton-Raphson method $[18,19]$ to find a numerical solution of this nonlinear equation when the shape parameter is known, i.e.,

$$
\widehat{\alpha}_{\mathrm{MLE}}=\left(\frac{\sum_{i=1}^{n} t_{i}^{\beta}}{n}\right)^{1 / \widehat{\beta}} \text {, }
$$

and

$$
\widehat{\beta}=\left[\left(\sum_{i=1}^{n} t_{i}^{\beta} \ln t_{i}\right)\left(\sum_{i=1}^{n} \hat{t}_{i}^{\beta}\right)^{-1}-\frac{1}{n} \sum_{i=1}^{n} \ln t_{i}\right]^{-1} .
$$


The obtained MLE estimates of $\alpha$ then $\beta$ are often biased when sample sizes are small, i.e., $(n<30)$. The MLE estimates become unbiased and achieve constancy property when the sample size is large [20]. Since the property of constancy characterizes the MLE, by using this property, we can get the MLE estimates of the reliability function, i.e.,

$$
\widehat{R}(t)=\exp \left[\frac{-t^{\hat{\beta}}}{\widehat{\alpha}}\right] .
$$

2.2.2. Method of Minimax (MOM). This estimation method primarily depends on Lemann's theorem; let $\left(\tau=F_{\alpha}, \alpha \in \Theta\right)$ be a family of distribution functions and $D$ be a class of estimators of $\alpha$. Suppose that $\left(\delta^{*} \in D\right)$ is a Bayes estimator against a prior distribution $\xi^{*}(\alpha)$ on $\Theta$, the risk function $R\left(\delta^{*}, \alpha\right)$ equals constant on $\Theta$, and $\delta^{*}$ is a minimax estimator [21]. Assuming a quadratic loss function $L=$ Loss $=\left(\alpha-\delta_{1} / \alpha\right)^{2}$, we can find the estimator $\left(\delta_{1}^{*}\right)$ as the initial distribution of the parameter $(\alpha)$ distributed as a gamma distribution [21, 22].

$$
\pi(\alpha)=\frac{1}{\Gamma(\theta) \beta^{\theta}} \alpha^{\theta-1} e^{-\alpha / \beta}, \quad, \alpha>0, \theta, \beta>0 .
$$

A formula is derived for the estimator $\left(\delta_{1}^{*}\right)$ of the parameter $(\alpha)$; after, it is found that the subsequent distribution of the parameter $(\alpha)$ with a random sample $X=\left(x_{1}, x_{2}, x_{3}, \ldots, x_{n}\right)$ is

$$
\begin{aligned}
\pi(\alpha \mid t)= & \frac{f(\alpha, t)}{f(t)}=\frac{(1 / \alpha)^{\theta+n+1} e^{-1 / \alpha\left(\sum_{i=1}^{n} t_{i}^{\beta}+1 / \lambda\right)}}{\int_{0}^{\infty}(1 / \alpha)^{\theta+n+1} e^{-\left(\sum_{i=1}^{n} t_{i}^{\beta} / \alpha+1 / \lambda \alpha\right)} \mathrm{d} \alpha}, \\
= & \frac{\left(\sum_{i=1}^{n} t_{i}^{\beta}+1 / \lambda\right)^{n+\theta}}{\Gamma(n+\theta)}\left(\frac{1}{\alpha}\right)^{\theta+n+1} e^{--1 / \alpha}\left(\sum_{i=1}^{n} t_{i}^{\beta}+1 / \lambda\right) \\
& \pi(\alpha \mid t) \sim \operatorname{Gamma}\left(n+\theta, \sum_{i=1}^{n} t_{i}^{\beta}+\frac{1}{\lambda}\right) .
\end{aligned}
$$

Depending on the quadratic loss function $\psi_{\varrho}^{\beta_{1}}=$ $\left(\alpha-\delta_{1}^{*} / \alpha\right)^{2}$,

$$
\begin{aligned}
\text { Loss } & =\left(\frac{\alpha-\delta_{1}}{\alpha}\right)^{2}, \\
R(\delta, \alpha) & =E[\text { Loss }] \\
R(\delta, \alpha) & =\pi(\alpha \mathrm{I} t)-2 \delta_{1} E\left(\frac{1}{\alpha}\right)+\delta_{1}^{2} E\left(\frac{1}{\alpha_{1}^{2}}\right), \\
\frac{\partial R(\delta, \alpha)}{\partial d} & =-2 E\left(\frac{1}{\alpha}\right)+2 \delta_{1} E\left(\frac{1}{\alpha_{1}^{2}}\right) \\
\Rightarrow \delta_{1}^{*} & =\frac{E(1 / \alpha \mid t)}{E\left(1 / \alpha_{1}^{2} \mid t\right)},
\end{aligned}
$$

where

$$
\begin{aligned}
E\left(\frac{1}{\alpha} \mid t\right) & =\int_{0}^{\infty} \frac{1}{\alpha} \pi(\alpha \mid t) \mathrm{d} \alpha=\frac{n+\theta}{\sum_{i=0}^{n} t_{i}^{\beta}+1 / \lambda} \\
\left(\frac{1}{\alpha_{1}^{2}} \mid t\right) & =\int_{0}^{\infty} \frac{1}{\alpha^{2}} \pi(\alpha \mid t) \mathrm{d} \alpha=\frac{(n+\theta)(n+\theta+1)}{\left(\sum_{i=0}^{n} t_{i}^{\beta}+1 / \lambda\right)^{2}}, \\
\delta_{1}^{*} & =\frac{E(1 / \alpha)}{E\left(1 / \alpha_{1}^{2}\right)}=\frac{\int_{0}^{\infty} 1 / \alpha \pi(\alpha \mathrm{I} t) \mathrm{d} \alpha}{\int_{0}^{\infty} 1 / \alpha^{2} \pi(\alpha \mathrm{I} t) \mathrm{d} \alpha} \\
& =\frac{\left(n+\theta / \sum_{i=0}^{n} t_{i}^{\beta}+1 / \lambda\right)}{(n+\theta)(n+\theta+1) /\left(\sum_{i=0}^{n} t_{i}^{\beta}+1 / \lambda\right)^{2}}, \\
\delta_{1}^{*} & =\frac{\sum_{i=0}^{n} t_{i}^{\beta}+1 / \lambda}{(n+\theta+1)}=\mathrm{M}_{1}\left(\eta+\frac{1}{\lambda}\right),
\end{aligned}
$$

where

$$
\begin{aligned}
\mathrm{M}_{1} & =\frac{1}{(n+\theta+1)}, \\
\eta & =\sum_{i=1}^{n} t_{i}^{\beta} .
\end{aligned}
$$

2.2.3. Modified Linear Exponential (MLINEX) Loss Function. It is a modified linear exponential loss function. It is frequently used in statistical estimation and prediction problems. It can be expressed as follows:

$$
L\left(\alpha, \delta_{2}\right)=k\left[\left(\frac{\delta_{2}}{\alpha}\right)^{c}-c \ln \left(\frac{\delta_{2}}{\alpha}\right)-1\right],
$$

where $\delta_{2}$ is the estimator of the parameter $(\alpha)$ and $k, c$ are constants in the loss function.

The estimator in minimax of the parameter $\alpha$ under the loss function (21) is $\delta_{2}$ which is symbolized as $\psi_{M L}^{\beta_{1}}$ :

$$
\begin{aligned}
\delta_{2} & =\psi_{M L}^{\beta_{1}}=\left[E\left(\alpha^{-c}\right)\right]^{-1 / c}, \\
E\left(\alpha^{-c}\right) & =\int_{0}^{\infty} \alpha^{-c} \pi(\alpha \mid t) \mathrm{d} \alpha, \\
& =\int_{0}^{\infty} \frac{\left(\sum_{i=1}^{n} t_{i}^{\beta}+1 / \lambda\right)^{n+\theta}}{\Gamma(n+\theta)} \alpha^{-(\theta+n+c+1)} e^{-1 / \alpha}\left(\sum_{i=1}^{n} t_{i}^{\beta}+1 / \lambda\right)_{\mathrm{d} \alpha} \\
& =\frac{\Gamma(\theta+n+c)}{\Gamma(\theta+n)}\left(\sum_{i=1}^{n} t_{i}^{\beta}+\frac{1}{\lambda}\right)^{-c} .
\end{aligned}
$$

The second estimator minimax under a modified quadratic loss function is

$$
\delta_{2}^{*}=\frac{\Gamma(\theta+n+c)}{\Gamma(\theta+n)}\left(\sum_{i=1}^{n} t_{i}^{\beta}+\frac{1}{\lambda}\right)^{-c}=\mathrm{M}_{2}\left(\eta+\frac{1}{\lambda}\right),
$$


where

$$
\begin{aligned}
\mathrm{M}_{2} & =\left(\frac{\Gamma(n+\theta)}{\Gamma(\theta+n+c)}\right)^{1 / c}, \\
\eta & =\sum_{i=1}^{n} t_{i}^{\beta} .
\end{aligned}
$$

2.2.4. White's Method. This method is mainly based on the cumulative distribution function presented in equation (2) to formulate a simple linear slope model as follows:

$$
\begin{aligned}
F\left(t_{i}\right) & =1-e^{-\left(t_{i} / \alpha\right)^{\beta}}, \\
1-F\left(t_{i}\right) & =e^{-\left(t_{i} / \alpha\right)^{\beta}}, \\
\frac{1}{1-F\left(t_{i}\right)} & =e^{\left(t_{i} / \alpha\right)^{\beta}} .
\end{aligned}
$$

Equation (25) can be linearized by taking the logarithm twice on both sides:

$$
\ln \left[-\ln \left(1-F\left(t_{i}\right)\right)\right]=\beta \ln t_{i}-\beta \ln \alpha .
$$

Now, we can write equation (26) as a linear regression model:

$$
Y_{i}=a+b T_{i}
$$

where

$$
\begin{aligned}
Y_{i} & =\ln \left[-\ln \left(1-F\left(t_{i}\right)\right)\right], \\
T_{i} & =\ln t_{i}, \\
a & =-\beta \ln \alpha, \text { and } b=\beta .
\end{aligned}
$$

Using the ordinary least squares method (OLSM), we can estimate the parameters of the regression model as follows:

$$
\begin{aligned}
\hat{b}_{L S} & =\frac{\sum_{i=1}^{n} T_{i} Y_{i}-n \overline{T Y}}{\sum_{i=1}^{n} T_{i}^{2}-\left(\sum_{i=1}^{n} T_{i}\right)^{2}}, \\
\widehat{a}_{\mathrm{OLS}} & =\bar{Y}-\widehat{b}_{L S} \bar{T},
\end{aligned}
$$

i.e.,

$$
\begin{aligned}
& \widehat{\alpha}=e^{-\widehat{a}_{L S},} \\
& \widehat{\beta}=\widehat{b}_{L S} .
\end{aligned}
$$

It can also be obtained by estimating the first order of the Taylor series for ascending order data as follows, assuming that

$$
\begin{aligned}
Z_{i} & =\ln \left(-\ln \left(1-p_{i}\right)\right), \\
t\left(\mathrm{P}_{i}\right) & =\beta+\alpha Z_{i}, \\
\frac{\partial t}{\partial \beta} & =1,
\end{aligned}
$$

$$
\begin{aligned}
\frac{\partial t}{\partial \alpha} & =Z_{i}, \\
f(a+b) & =f(a)+h_{1} f^{\prime}(a)+h_{2} f(a), \\
\therefore f(a) & =\beta_{0}+\alpha_{0} Z_{i}, \\
E\left(t_{i}\right) & =\beta_{0}+\alpha_{0} Z_{i}+\left(\beta-\beta_{0}\right) \cdot 1+\left(\theta-\theta_{0}\right) Z_{i}, \\
& =\beta_{0}+\alpha_{0} Z_{i}+\beta-\beta_{0}+\theta Z_{i}-\theta_{0} Z_{i}=\beta+\theta Z_{i} .
\end{aligned}
$$

By using the method of least squares, dependent on the matrix approach, we can estimate the parameters of the Weibull distribution by extracting the estimated values of $\alpha, \beta$ from the following formula:

$$
\left[\begin{array}{l}
\widehat{\beta} \\
\widehat{\alpha}
\end{array}\right]=\left(H^{T} H\right)^{-1} H^{T} t\left(Z_{i}\right),
$$

where

$$
H=\left[\begin{array}{cc}
1 & z_{1} \\
1 & z_{2} \\
\vdots & \vdots \\
1 & z_{n}
\end{array}\right]
$$

\section{Results and Discussion}

3.1. The Comparison. The efficiency of estimation methods can be compared by comparing the variance of estimation methods. For comparing the efficiency of the two estimators $\left(\delta_{1}^{*}, \delta_{2}^{*}\right)$, we need to calculate the variance of each one of them separately:

$$
\begin{aligned}
& \operatorname{Var}\left(\delta_{1}^{*}\right)=\frac{1}{(\theta+n+1)^{2}} \operatorname{Var}\left(\sum_{i=1}^{n} t_{i}^{\beta}+\frac{1}{\lambda}\right), \\
& \operatorname{Var}\left(\delta_{2}^{*}\right)=\left(\frac{\Gamma(\theta+n)}{\Gamma(\theta+n+c)}\right)^{2 / c} \operatorname{Var}\left(\sum_{i=1}^{n} t_{i}^{\beta}+\frac{1}{\lambda}\right) .
\end{aligned}
$$

The efficiency of $\delta_{2}^{*}$ relative to $\delta_{1}^{*}$ is as follows:

$$
\text { eff } \begin{aligned}
\left(\delta_{1}^{*}, \delta_{2}^{*}\right) & =\frac{(\Gamma(\theta+n) / \Gamma(\theta+n+c))^{2 / c} \operatorname{Var}\left(\sum_{i=1}^{n} t_{i}^{\beta}+1 / \lambda\right)}{1 /(\theta+n+1)^{2} \operatorname{Var}\left(\sum_{i=1}^{n} t_{i}^{\beta}+1 / \lambda\right)} \\
& =\frac{(\Gamma(\theta+n) / \Gamma(\theta+n+c))^{2 / c}}{1 /(\theta+n+1)^{2}} .
\end{aligned}
$$

When $c=1$, it becomes

$$
\text { eff }\left(\delta_{1}^{*}, \delta_{2}^{*}\right)=\frac{(\theta+n+1)^{2}}{(\theta+n)^{2}}>1 \text {. }
$$

When $c=-1$, it becomes

$$
\text { eff }\left(\delta_{1}^{*}, \delta_{2}^{*}\right)=\frac{(\theta+n+1)^{2}}{(\theta+n-1)^{2}}>1 \text {. }
$$


Since eff $>1$, the Bayes estimator under the quadratic loss function $\delta_{1}^{*}$ is more efficient than $\delta_{2}^{*}$.

To compare the efficiency of the estimators $\left(\delta_{1}^{*}, \widehat{\alpha}_{\mathrm{MLE}}\right)$, we need to calculate the variance of each estimator, i.e.,

$$
\operatorname{Var}\left(\widehat{\alpha}_{\mathrm{MLE}}\right)=\operatorname{Var}\left(\frac{\sum_{i=1}^{n} t_{i}^{\beta}}{n}\right)^{1 / \widehat{\beta}}=\frac{1}{\left(n^{1 / \beta}\right)^{2}} \operatorname{Var}\left(\sum_{i=1}^{n} t_{i}^{\beta}\right)^{1 / \beta} .
$$

Let $\lambda$ in equations (34) and (35) be constant and known then:

$$
\begin{aligned}
\operatorname{Var}\left(\delta_{1}^{*}\right) & =\frac{1}{(\theta+n+1)^{2}} \operatorname{Var}\left(\sum_{i=1}^{n} t_{i}^{\beta}\right), \\
\operatorname{Var}\left(\delta_{2}^{*}\right) & =\left(\frac{\Gamma(\theta+n)}{\Gamma(\theta+n+c)}\right)^{2 / c} \operatorname{Var}\left(\sum_{i=1}^{n} t_{i}^{\beta}\right), \\
\operatorname{Var}\left(\widehat{\alpha}_{\text {OLS }}\right) & =\frac{\widehat{b}_{\text {OLS }}^{2}}{n^{2}} \operatorname{Var}\left(\sum_{i=1}^{n} t_{i}\right) .
\end{aligned}
$$

Thus, the efficiency of $\delta_{1}^{*}$ relative to $\widehat{\alpha}_{\mathrm{MLE}}$ equals

$$
\begin{aligned}
& \operatorname{eff}\left(\widehat{\alpha}_{\mathrm{MLE}}, \delta_{1}^{*}\right)=\frac{1 /(\theta+n+1)^{2} \operatorname{Var}\left(\sum_{i=1}^{n} t_{i}^{\beta}\right)}{1 /\left(n^{1 / \beta}\right)^{2} \operatorname{Var}\left(\sum_{i=1}^{n} t_{i}^{\beta}\right)^{1 / \beta}}>1, \\
& \operatorname{eff}\left(\widehat{\alpha}_{\mathrm{MLE}}, \delta_{2}^{*}\right)=\frac{(\Gamma(\theta+n) / \Gamma(\theta+n+c))^{2 / c} \operatorname{Var}\left(\sum_{i=1}^{n} t_{i}^{\beta}\right)}{1 /\left(n^{1 / \beta}\right)^{2} \operatorname{Var}\left(\sum_{i=1}^{n} t_{i}^{\beta}\right)^{1 / \beta}}>1 .
\end{aligned}
$$

Since eff $>1$, the efficiency of the maximum likelihood estimator $\widehat{\alpha}_{\mathrm{MLE}}$ is more efficient than the estimators $\delta_{1}^{*}$ and $\delta_{2}^{*}$.

The efficiency of $\widehat{\alpha}_{\mathrm{MLE}}$ relative to $\widehat{\alpha}_{\mathrm{OLS}}$ equals

$$
\text { eff }\left(\widehat{\alpha}_{\mathrm{MLE}}, \widehat{\alpha}_{\mathrm{OLS}}\right)=\frac{\widehat{b}_{\mathrm{OLS}}^{2} / n^{2} \operatorname{Var}\left(\sum_{i=1}^{n} t_{i}\right)}{1 /\left(n^{1 / \beta}\right)^{2} \operatorname{Var}\left(\sum_{i=1}^{n} t_{i}^{\beta}\right)^{1 / \beta}}>1 \text {, }
$$

and hence $\widehat{\alpha}_{\text {MLE }}$ is more efficient than $\widehat{\alpha}_{\text {OLS }}$.

From the above mathematical expressions, it can be concluded the maximum likelihood estimator $\widehat{\alpha}_{\text {MLE }}$ is more efficient than $\delta_{1}^{*}, \delta_{2}^{*}$, and $\widehat{\alpha}_{\text {OLS }}$ in the scale parameter estimation for the Weibull distribution. Also, in the case of Bayes estimator under the quadratic loss function, $\delta_{1}^{*}$ is more efficient than $\delta_{2}^{*}$.

3.2. Simulation. The simulation technique was used to achieve the objective of the study, to compare the estimation methods experimentally (maximum likelihood, minimax, MLINEX, and White's method). This method is characterized by flexibility, and to achieve this purpose, random samples were generated for the parameters to be estimated using the Monte Carlo method theoretically, not practically, and without violating the accuracy of the results required according to the following steps:
(1) The assumed values for the real distribution parameters were determined by Table 1 from four different models, including different values for the shape and scale parameters.

(2) The assumed four different sizes were identified for sample $(n=10,50,100,150)$ and frequency of experiment $(L=500,1000,2000) .{ }^{*} \mathrm{C}$ is the Jeffrey constant.

(3) The data are generated from the Weibull distribution for assumed values of parameters and specified sample sizes, i.e.,

(1) Generating random numbers $V_{i}$ that follow the uniform distribution on the interval $(0,1)$.

$$
U_{i} \sim U(0,1), \quad i=1,2, \ldots, n,
$$

where $U_{i}$ is a continuous random variable.

(2) Conversion of the data generated in Step (1), which follows the uniform distribution to the Weibull distribution data using the cumulative distribution function, according to the following inverse conversion method:

$$
\begin{aligned}
F(t) & =1-e^{-\left(t_{i} / \alpha\right)^{\beta}}, \\
1-F(t) & =e^{-\left(t_{i} / \alpha\right)^{\beta}}, \\
1-U & =e^{-\left(t_{i} / \alpha\right)^{\beta}}, \\
\ln (1-U) & =-\left(\frac{t_{i}}{\alpha}\right)^{\beta}=\frac{-t^{\beta}}{\alpha^{\beta}}, \\
t & =\left[-\alpha^{\beta} \ln (1-U)\right]^{\beta}, \quad 0 \leq U \leq 1 .
\end{aligned}
$$

\section{Comparison of Estimation Methods}

The mean square error (MSE) was considered as a basis for comparison between estimation methods, which takes the following form:

$$
\operatorname{MSE}(\widehat{\alpha})=\frac{\sum_{i=1}^{L}\left(\widehat{\alpha}_{i}-\alpha\right)^{2}}{L},
$$

where $L$ is the frequency of each experiment.

\section{Results}

The results of simulation experiments were presented and interpreted to find the best estimate of distribution parameters by applying equations (13), (19), (23), and (30). The experiment was carried out on values generated from equation (44) to find the efficiency of the estimators using MATLAB 15 in the generation process, as shown in Table 2.

Table 2 shows that the maximum likelihood method was the best and more efficient in estimating the scale parameter 
TABLE 1: Different values of the parameters used.

\begin{tabular}{lccccc}
\hline Model & $\alpha$ & $\beta$ & $\lambda$ & $\theta$ & $C^{*}$ \\
\hline I & 0.5 & 0.6 & 1 & 0.5 & 0.5 \\
II & 0.7 & 0.8 & 1 & 1 & -1 \\
III & 1.0 & 0.9 & 0.5 & 1 & 2 \\
IV & 1.3 & 1.2 & 0.5 & -2 \\
\hline
\end{tabular}

TABLe 2: The MSE values for all methods and sample sizes used in simulations when $L=500$.

\begin{tabular}{|c|c|c|c|c|c|c|c|c|c|c|}
\hline \multirow{2}{*}{ Model } & \multirow{2}{*}{$N$} & \multicolumn{4}{|c|}{ MSE } & \multicolumn{4}{|c|}{ Priority order of methods } & \multirow{2}{*}{ Best } \\
\hline & & MLINEX & MOM & MLE & OLS & MLINEX & MOM & MLE & OLS & \\
\hline \multirow{4}{*}{ I } & 10 & 0.0904 & 0.0531 & 0.0731 & 1.8971 & 3 & 1 & 2 & 4 & MOM \\
\hline & 50 & 0.0391 & 0.0355 & 0.0138 & 0.9512 & 3 & 2 & 1 & 4 & MLE \\
\hline & 100 & 0.0306 & 0.0294 & 0.0064 & 0.8214 & 3 & 2 & 1 & 4 & MLE \\
\hline & 150 & 0.0297 & 0.0278 & 0.0043 & 0.0984 & 3 & 2 & 1 & 4 & MLE \\
\hline \multirow{4}{*}{ II } & 10 & 0.1446 & 0.0639 & 0.0869 & 2.1457 & 3 & 1 & 2 & 4 & MOM \\
\hline & 50 & 0.0432 & 0.0317 & 0.0139 & 0.8742 & 3 & 2 & 1 & 4 & MLE \\
\hline & 100 & 0.0341 & 0.0299 & 0.0079 & 0.0657 & 3 & 2 & 1 & 4 & MLE \\
\hline & 150 & 0.0324 & 0.0283 & 0.0053 & 0.7421 & 3 & 2 & 1 & 4 & MLE \\
\hline \multirow{4}{*}{ III } & 10 & 0.0929 & 0.0265 & 0.0865 & 1.6971 & 3 & 1 & 2 & 4 & MOM \\
\hline & 50 & 0.0401 & 0.0382 & 0.0184 & 0.8145 & 3 & 2 & 1 & 4 & MLE \\
\hline & 100 & 0.0348 & 0.0314 & 0.0096 & 0.0985 & 3 & 2 & 1 & 4 & MLE \\
\hline & 150 & 0.0311 & 0.0296 & 0.0061 & 0.0745 & 3 & 2 & 1 & 4 & MLE \\
\hline \multirow{4}{*}{ IV } & 10 & 0.2049 & 0.0818 & 0.1154 & 0.8742 & 3 & 1 & 2 & 4 & MOM \\
\hline & 50 & 0.0504 & 0.0367 & 0.0241 & 0.0954 & 3 & 2 & 1 & 4 & MLE \\
\hline & 100 & 0.0381 & 0.0312 & 0.0116 & 0.0512 & 3 & 2 & 1 & 4 & MLE \\
\hline & 150 & 0.0343 & 0.0300 & 0.0081 & 0.0451 & 3 & 2 & 1 & 4 & MLE \\
\hline
\end{tabular}

for Weibull distribution, with the lowest MSE for all models and sample sizes used, compared with other methods, except small sample sizes $n=10$. On the other hand, the minimax method based on the quadratic loss function was the most efficient for all models; it can be observed from the results of the last column of Table 2. Hence, the maximum likelihood method was the best in 12 out of the 16 estimations for the scale parameter.

Also, it can be observed that the MSE values decreased with the increase in the sample size for all models. It corresponds to the statistical theory, which confirms the validity of the theoretical side in this paper about the behavior of this function. The simulation results are similar, although the experiment was repeated 500, 1000, and 2000 times, which shows the estimators' stability and efficiency.

The simulation results showed that the MOM method ranked second in the efficiency in the case of medium and large samples and for all models and sizes of samples. The MLINEX ranked third in terms of efficiency, and OLS ranked fourth with the lowest efficiency. Likewise, we obtained the same results in Tables 3 and 4 .

Table 3 also shows that the maximum likelihood method was the best and more efficient in estimating the scale parameter for Weibull distribution, with the lowest MSE for all models and sample sizes used, compared with other methods, except small sample sizes $n=10$. The minimax method based on the quadratic loss function was the most efficient for all models. It is clear in the results of the last column of Table 3. Also, it is observed that the MSE values decreased with the increase in the sample size for all models. This, however, corresponds to the statistical theory. The simulation results are similar, although the experiment was repeated 500, 1000, and 2000 times, and this shows the stability of the estimator's efficiency.

The simulation results showed that the MOM method ranked second in the efficiency in the case of medium and large samples and for all models and sizes of samples. The MLINEX ranked third in terms of efficiency, and OLS ranked fourth with the lowest efficiency.

Likewise, Table 4 also shows that the maximum likelihood method was the best and more efficient in estimating the scale parameter for Weibull distribution, with the lowest MSE for all models and sample sizes used, compared with other methods except small sample sizes $n=10$.

The minimax method based on the quadratic loss function was the most efficient for all models. It is clear in the results of the last column of Table 4 , and hence the maximum likelihood method was the best in 12 of the total 16 estimations for the scale parameter.

Also, it can be observed that the MSE values decreased with the increase in the sample size for all models. The simulation results are similar, although the experiment was repeated 500, 1000, and 2000 times, and this shows the stability of the efficiency of the estimators. The simulation results showed that the MOM method ranked second in the efficiency in the case of medium and large samples and for all models and sizes of samples. The MLINEX ranked third in terms of efficiency, and OLS ranked fourth with the lowest efficiency. 
TABLE 3: The MSE values for all methods and sample sizes used in simulations when $L=1000$.

\begin{tabular}{|c|c|c|c|c|c|c|c|c|c|c|}
\hline \multirow{2}{*}{ Model } & \multirow{2}{*}{$\mathrm{N}$} & \multicolumn{4}{|c|}{ MSE } & \multicolumn{4}{|c|}{ Priority order of methods } & \multirow{2}{*}{ Best } \\
\hline & & MLINEX & $\mathrm{MOM}$ & MLE & OLS & MLINEX & MOM & MLE & OLS & \\
\hline \multirow{4}{*}{ I } & 10 & 0.0946 & 0.0572 & 0.0731 & 2.1645 & 3 & 1 & 2 & 4 & MOM \\
\hline & 50 & 0.0391 & 0.0338 & 0.0142 & 1.1245 & 3 & 2 & 1 & 4 & MLE \\
\hline & 1600 & 0.0324 & 0.0288 & 0.0074 & 0.9542 & 3 & 2 & 1 & 4 & MLE \\
\hline & 150 & 0.0294 & 0.0283 & 0.0046 & 0.8421 & 3 & 2 & 1 & 4 & MLE \\
\hline \multirow{4}{*}{ II } & 10 & 0.1341 & 0.0590 & 0.0802 & 2.2541 & 3 & 1 & 2 & 4 & MOM \\
\hline & 50 & 0.0410 & 0.0338 & 0.0146 & 1.1987 & 3 & 2 & 1 & 4 & MLE \\
\hline & 100 & 0.0741 & 0.0392 & 0.0282 & 0.8741 & 3 & 2 & 1 & 4 & MLE \\
\hline & 150 & 0.0311 & 0.0284 & 0.0056 & 0.0415 & 3 & 2 & 1 & 4 & MLE \\
\hline \multirow{4}{*}{ III } & 10 & 0.0949 & 0.0780 & 0.0889 & 2.0245 & 3 & 1 & 2 & 4 & MOM \\
\hline & 50 & 0.0401 & 0.0385 & 0.0193 & 1.7951 & 3 & 2 & 1 & 4 & MLE \\
\hline & 100 & 0.0326 & 0.0318 & 0.0099 & 1.6281 & 3 & 2 & 1 & 4 & MLE \\
\hline & 150 & 0.0315 & 0.0288 & 0.0069 & 0.0782 & 3 & 2 & 1 & 4 & MLE \\
\hline \multirow{4}{*}{ IV } & 10 & 0.2112 & 0.0744 & 0.1190 & 2.3547 & 3 & 1 & 2 & 4 & MOM \\
\hline & 50 & 0.0938 & 0.0372 & 0.0229 & 1.8416 & 3 & 2 & 1 & 4 & MLE \\
\hline & 100 & 0.0397 & 0.0324 & 0.0118 & 0.6874 & 3 & 2 & 1 & 4 & MLE \\
\hline & 150 & 0.0344 & 0.0291 & 0.0079 & 0.0746 & 3 & 2 & 1 & 4 & MLE \\
\hline
\end{tabular}

TABLE 4: The MSE values for all methods and sample sizes used in simulations when $L=2000$.

\begin{tabular}{|c|c|c|c|c|c|c|c|c|c|c|}
\hline \multirow{2}{*}{ Model } & \multirow{2}{*}{$\mathrm{N}$} & \multicolumn{4}{|c|}{ MSE } & \multicolumn{4}{|c|}{ Priority order of methods } & \multirow{2}{*}{ Best } \\
\hline & & MLINEX & MOM & MLE & OLS & MLINEX & MOM & MLE & OLS & \\
\hline \multirow{4}{*}{ I } & 10 & 0.0908 & 0.0610 & 0.0753 & 2.4512 & 3 & 1 & 2 & 4 & MOM \\
\hline & 50 & 0.0390 & 0.0321 & 0.0151 & 1.2571 & 3 & 2 & 1 & 4 & MLE \\
\hline & 100 & 0.0320 & 0.0308 & 0.0067 & 1.0578 & 3 & 2 & 1 & 4 & MLE \\
\hline & 150 & 0.0306 & 0.0293 & 0.0052 & 0.0743 & 3 & 2 & 1 & 4 & MLE \\
\hline \multirow{4}{*}{ II } & 10 & 0.1321 & 0.0583 & 0.0784 & 1.9845 & 3 & 1 & 2 & 4 & MOM \\
\hline & 50 & 0.0432 & 0.0324 & 0.0156 & 1.1234 & 3 & 2 & 1 & 4 & MLE \\
\hline & 100 & 0.0347 & 0.0299 & 0.0087 & 1.0451 & 3 & 2 & 1 & 4 & MLE \\
\hline & 150 & 0.0310 & 0.0291 & 0.0053 & 0.6548 & 3 & 2 & 1 & 4 & MLE \\
\hline \multirow{4}{*}{ III } & 10 & 0.0927 & 0.0740 & 0.0873 & 1.8257 & 3 & 1 & 2 & 4 & $\mathrm{MOM}$ \\
\hline & 50 & 0.0403 & 0.0373 & 0.0205 & 1.0871 & 3 & 2 & 1 & 4 & MLE \\
\hline & 100 & 0.0341 & 0.0324 & 0.0099 & 0.8246 & 3 & 2 & 1 & 4 & MLE \\
\hline & 150 & 0.0311 & 0.0298 & 0.0074 & 0.0687 & 3 & 2 & 1 & 4 & MLE \\
\hline \multirow{4}{*}{ IV } & 10 & 0.2091 & 0.0804 & 0.1209 & 1.1248 & 3 & 1 & 2 & 4 & MOM \\
\hline & 50 & 0.0923 & 0.0382 & 0.0225 & 1.0835 & 3 & 2 & 1 & 4 & MLE \\
\hline & 100 & 0.0377 & 0.0318 & 0.0125 & 0.5413 & 3 & 2 & 1 & 4 & MLE \\
\hline & 150 & 0.0344 & 0.0289 & 0.0081 & 0.0579 & 3 & 2 & 1 & 4 & MLE \\
\hline
\end{tabular}

\section{Discussion}

The present analysis is commenced to select an appropriate parameter estimation method among several estimation methods for two-parameter Weibull distribution. Generally, the maximum likelihood method is used for parameter estimation when the shape parameter is known. However, we estimated mathematically the scale parameter of the twoparameter Weibull distribution. The relative efficient estimation method is identified based on minimum MSE. The theoretical outcomes demonstrated that the efficiency of the obtained estimator $\widehat{\alpha}_{\text {MLE }}$ from MLE is more efficient than the estimators $\delta_{1}^{*}, \delta_{2}^{*}$, and $\widehat{\alpha}_{\text {OLS }}$ applied in estimation for the parameters. Further, the results demonstrated that the estimator obtained from MLE is best and more efficient in estimating the scale parameter than other associated methods. Furthermore, increasing the sample size reduced the MSE. Further, the acquired information validated that simulation results are providing the same information as hypothetical results.

\section{Conclusion}

The theoretical results showed that the efficiency of the maximum likelihood estimator $\widehat{\alpha}_{\text {MLE }}$ is more efficient than the estimators $\delta_{1}^{*}, \delta_{2}^{*}$, and $\widehat{\alpha}_{\text {OLS }}$ used in estimation for the parameters of Weibull distribution. Further, the results show that the maximum likelihood estimator is best and more efficient in estimating the scale parameter than the other methods. Moreover, the mean squares error (MSE) was reduced with the increase in the size of the sample. Furthermore, the theoretical results are validated by the simulation results. The obtained information validates that simulation results are providing the same information as hypothetical results. However, the experiment was repeated 500, 1000, and 2000 times to show the stability of the 
efficiency of the estimators. The maximum likelihood method ranked first, the minimax method ranked second, MLINEX ranked third, and OLS ranked fourth in terms of efficiency.

\section{Data Availability}

The data used to support the theoretical findings were generated via simulation using MATLAB 15.

\section{Conflicts of Interest}

The authors declare that they have no conflicts of interest.

\section{Acknowledgments}

The authors extend their appreciation to the Deanship of Scientific Research at King Khalid University for funding this work (Grant no. RGP. 1/26/42 to Mohammed M. Almazah).

\section{References}

[1] O. Corzo, N. Bracho, A. Pereira, and A. Vásquez, "Weibull distribution for modeling air drying of coroba slices," Lebensmittel-Wissenschaft und -Technologie- Food Science and Technology, vol. 41, no. 10, pp. 2023-2028, 2008.

[2] C. D. Lai, D. N. Murthy, and M. Xie, Weibull Distributions and Their ApplicationsSpringer Handbooks, Berlin, Germany, 2006.

[3] M. Mohammed, A. Al, S. Parkinson, A. P. Longstaff, and S. Fletcher, "Efficiency of the reliability function and scheduling method in determining the optimal preventive maintenance time for digital X-ray devices," vol. 9, no. 2, pp. 53-58, 2019.

[4] A. A. Bartolucci, K. P. Singh, A. D. Bartolucci, and S. Bae, "Applying medical survival data to estimate the three-parameter Weibull distribution by the method of probabilityweighted moments," Mathematics and Computers in Simulation, vol. 48, no. 4-6, 1999.

[5] H. Rinne, The Weibull Distribution, Taylor Francis, NY, USA, 1st edition, 2008.

[6] R. Jiang and D. N. P. Murthy, "A study of weibull shape parameter: properties and significance," Reliability Engineering and System Safety, vol. 96, no. 12, pp. 1619-1626, 2011.

[7] G. Muraleedharan, A. D. Rao, P. G. Kurup, N. U. Nair, and M. Sinha, "Modified Weibull distribution for maximum and significant wave height simulation and prediction," Coastal Engineering, vol. 54, no. 8, 2007.

[8] M. Teimouri, S. M. Hoseini, and S. Nadarajah, "Comparison of estimation methods for the weibull distribution," A Journal of Theoretical and Applied Statistics, vol. 47, no. 1, 2013.

[9] A. J. Hallinan, "A review of the weibull distribution," Journal of Quality Technology, vol. 25, no. 2, 1993.

[10] R. Coleman, N. L. Johnson, S. Kotz, and N. Balakrishnan, "Continuous univariate distributions," Journal of the Royal Statistical Society. Series A, vol. 159, no. 2, 1996.

[11] F. G. Akgül and B. Şenoğlu, Comparison of Estimation Methods for Inverse Weibull Distribution, in Proceedings of the LINSTAT2016 meeting, Istanbul, Turkey, August 2016.

[12] C. Chatfield, N. L. Johnson, S. Kotz, and N. Balakrishnan, "Continuous univariate distributions," Journal of The Royal Statistician, vol. 44, no. 4, pp. 544-545, 1995.
[13] A. Genc, M. Erisoglu, A. Pekgor, G. Oturanc, A. Hepbasli, and K. Ulgen, "Estimation of wind power potential using weibull distribution," Energy Sources, vol. 27, no. 9, 2005.

[14] J. H. Heo, J. D. Salas, and K. D. Kim, "Estimation of confidence intervals of quantiles for the Weibull distribution," Stochastic Environmental Research and Risk Assessment, vol. 15 , pp. 284-309, 2001.

[15] C. König and R. van de Schoot, "Bayesian statistics in educational research: a look at the current state of affairs," Educational Review, vol. 70, no. 4, 2018.

[16] J. K. Kruschke, H. Aguinis, and H. Joo, "The time has come: bayesian methods for data analysis in the organizational sciences," Organizational Research Methods, vol. 15, no. 4, pp. 722-752, 2012.

[17] C. Rietbergen, T. P. A. Debray, I. Klugkist, K. J. M. Janssen, and K. G. M. Moons, "Reporting of Bayesian analysis in epidemiologic research should become more transparent," Journal of Clinical Epidemiology, vol. 86, no. 2, pp. 51-58, 2017.

[18] N. R. Mann, "Point and interval estimation procedures for the two-parameter weibull and extreme-value distributions," Technometrics, vol. 10, no. 2, 1968.

[19] S. K. Singh, U. Singh, and D. Kumar, "Bayesian estimation of parameters of inverse Weibull distribution," Journal of Applied Statistics, vol. 40, no. 7, 2013.

[20] K. S. Sultan, N. H. Alsadat, and D. Kundu, "Bayesian and maximum likelihood estimations of the inverse Weibull parameters under progressive type-II censoring," Journal of Statistical Computation and Simulation, vol. 84, no. 10, 2014.

[21] M. K. Roy, C. K. Podder, and K. J. Bhuiyan, "Minimax estimation of the scale parameter of the Weibull distribution for quadratic and MLINEX loss functions," Jahangirnagar University Journal of Science, vol. 25, pp. 277-285, 2002.

[22] A. Mehrsai, H. R. Karimi, K. D. Thoben, and B. Scholz-Reiter, "Application of learning pallets for real-time scheduling by the use of radial basis function network," Neurocomputing, vol. 101, no. 3, pp. 82-93, 2013. 